\title{
Periodontal management of phenytoin induced gingival enlargement: a case report
}

\begin{abstract}
Gingival enlargement is known side effect of certain medications like anticonvulsants, calcium channel blockers and immunosuppressant. Phenytoin is one of the most common drugs associated with gingival overgrowth. The present case report describes a successful management of phenytoin induced gingival enlargement in 13 year old female by combined surgical and nonsurgical approach. This patient should be managed in systematic manner including oral prophylaxis, substitution of drug and surgical therapy if needed and supportive periodontal therapy. Present case showed no recurrence 1 year postoperative.
\end{abstract}

Keywords: drug induced, gingival hyperplasia, hypertrophy
Volume 8 Issue I - 2017

\author{
Namita Gupta,' Lata Goyal,' ND Gupta' \\ 'Department of Periodontics and Community Dentistry, Aligarh \\ Muslim University, India \\ ${ }^{2}$ Department of Dentistry,All India Institute of Medical Sciences, \\ India \\ Correspondence: Lata Goyal, Senior Research Associate, \\ Department of Dentistry, All India Institute of Medical Sciences, \\ Rishikesh, Uttarakhand, India, Email latagoyal83@gmail.com
}

Received: June 02, 2017 | Published: August 21, 2017

\section{Introduction}

Gingival enlargement or gingival overgrowth is the abnormal growth of periodontal tissues. Drug induced gingival enlargement is one of the most common cause of enlarged gingiva. Drugs associated with gingival enlargement are divided in to three major groups namely anticonvulsants, immunosuppressant and calcium channel blockers. ${ }^{1}$ Among the anticonvulsants, phenytoin is most commonly associated with gingival enlargement, with a prevalence rate of $50 \%$, although different authors have reported incidences from 3\% to $84.5 \%{ }^{2,3}$ Dilantin is a hydantoin, introduced by Merritt \& Putnam in 1938 for the treatment of all forms of epilepsy, except the petit mal. ${ }^{4} \mathrm{Kimball}$ in 1939 was the first to report drug associated gingival enlargement with the chronic use of antiepileptic drug phenytoin, ${ }^{3}$ imbalance in collagen degradation, rather than an increase in collagen synthesis has been suggested for its etiology. ${ }^{5}$ A possible relationship between tumor necrosis factor-alpha (TNF-a) production and phenytoin in human gingival fibroblasts has also been suggested. ${ }^{6}$ The host's response to pathogens associated with the biofilm may also play a role. Excessive gingival overgrowth itself can also change the gingival contour, impeding oral function and speech and having an anti-aesthetic effect. ${ }^{7}$ Additionally, it can also compromise effective oral hygiene and may have negative implications for the systemic health of affected patients. Several approaches for the treatment of gingival overgrowth including oral prophylaxis, nonsurgical and surgical (gingivectomy or a flap procedure) have been proposed. ${ }^{8}$ The aim of this report is to present a case of gingival enlargement due to phenytoin with combined nonsurgical and surgical approach.

\section{Case presentation}

A 13 year female patient was referred to the Department of Periodontics at Dr. Ziauddin Ahmad Dental College, Aligarh with the chief complaints of swelling of gums for the past 8-9 months. She felt very uncomfortable as the swelling interfered while chewing. She had history of generalized tonic clonic seizures due to lesion in left parietal lobe for the last 1 year and was on medication Tab phenytoin (300mg) once a day, Tab clobazam 10mg HS, Tab Folic acid 5mg once a day for last 1 year. Intraoral examination revealed, massive gingival overgrowth on the labial aspect of the upper and lower teeth (Figures $1 \& 2)$. Her oral hygiene was poor and bleeding on probing was also present. Routine blood and orthopantomographical examination were within normal limit (Figure 3). Phenytoin was omitted as per advice of physician and then switching over to valproic acid. Full mouth scaling and polishing of all teeth was done and patient was given oral hygiene instruction Review after four weeks revealed some reduction of the gingival overgrowth particularly at the upper arch. At the following visit, surgical gingivectomy was performed for the lower enlarged gingiva. The overgrown tissue was resected by using scalpel blade size 15. After 1 week later the same procedure was done for the upper gingival overgrowth. All procedures were carried out under local anaesthesia and antibiotic coverage. Histopathological examination (HPE) reveals a pronounced hyperplasia of the connective tissue and epithelium. There is acanthosis of the epithelium and elongated rete-pegs extending deep into the connective tissue, which exhibits densely arranged collagen bundles, along with an increase in new blood vessels and fibroblasts (Figure 4). Patient was prescribed mouthwash chlorhexidine gluconate $0.12 \%$ for two weeks after each surgical procedure. Healing procedure was uneventful. On seventh day of follow up visit periodontal pack was removed. There was no recurrence till one year follow up (Figure 5\&6).

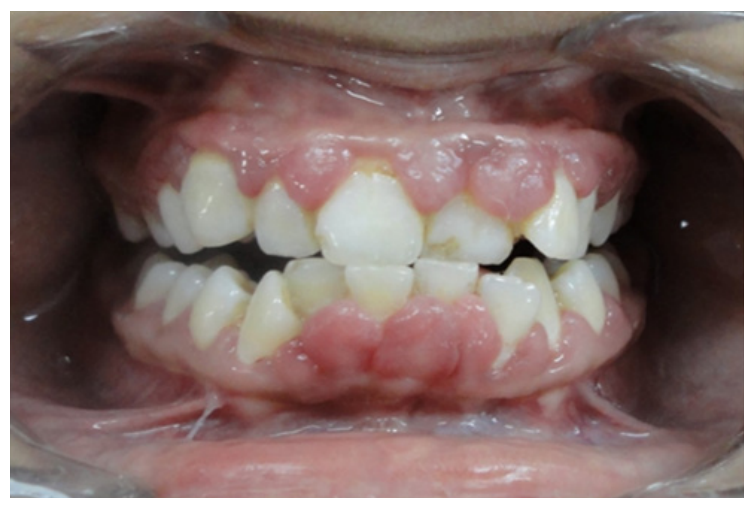

Figure I Preoperative labial view showing gingival enlargement in interdental and marginal gingiva. 


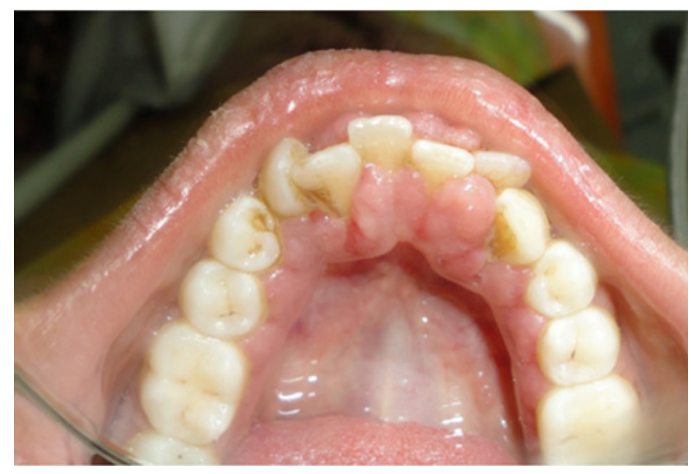

Figure 2 lingual view showing gingival enlargement.

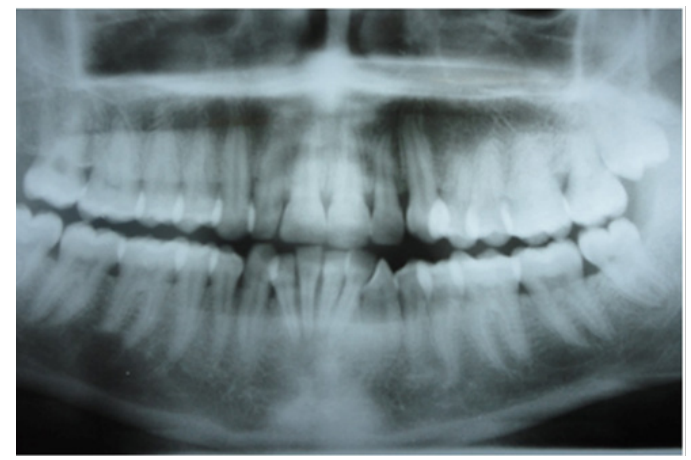

Figure 3 OPG showing normal bone pattern.

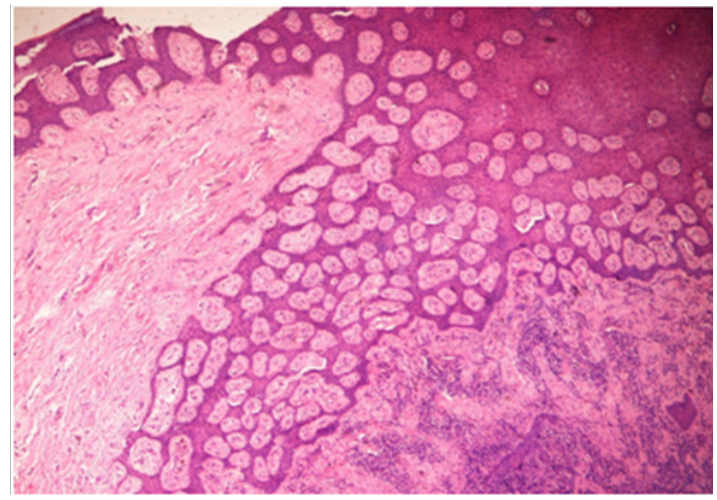

Figure 4 Histopathological examination showing acanthosis, elongated rete pegs and fibroblasts.

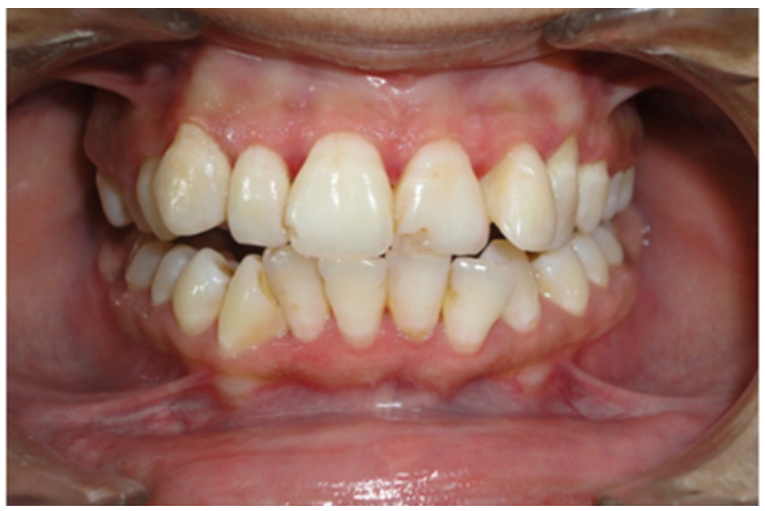

Figure 5 Postoperative labial view.

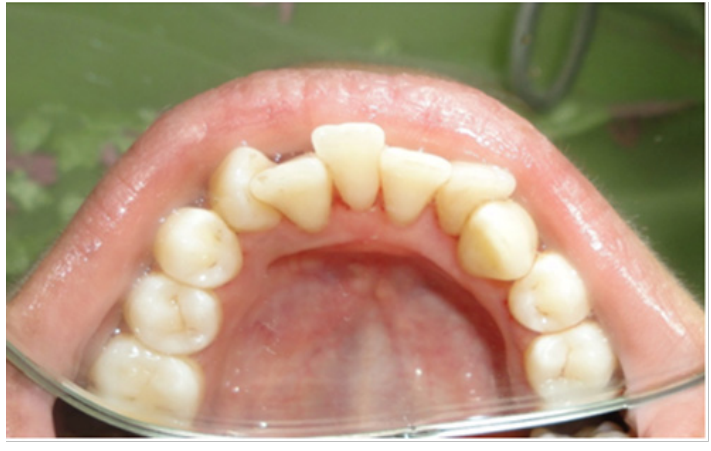

Figure 6 Postoperative lingual view.

\section{Discussion}

Phenytoin is the drug of choice for treatment for grand mal, temporal lobe, and psychomotor seizures since it was first introduced in the 1930s. ${ }^{4}$ The first reported cases of gingival enlargement as a result of PHT therapy appeared soon after the drug was introduced. ${ }^{3}$ The gingival growth usually begins as a diffuse swelling of the interdental papillae, which enlarge and coalesce, leaving a nodular appearance. The clinical and microscopic appearance of drug induced gingival enlargement is similar with any drug, although mechanism of action may be different. Several factors like age, genetic susceptibility, histopathology, tissue homeostasis may influence the relationship between the drugs and gingival tissues. ${ }^{3}$ There is increased protein and collagen production by subset of fibroblastin phenytoin induced gingival enlargement. ${ }^{9}$ Others have suggested a role of increase in non-collagenous component and a lack of collagen breakdown ${ }^{10}$ a role for intracellular calcium $\left(\mathrm{Ca}^{++}\right)$and folate deficiency in the pathogenesis of the gingival overgrowth has also been suggested. ${ }^{11,12}$ Studies show an association between the oral hygiene status and the severity of drug induced gingival enlargement. This suggests that plaque-induced gingival inflammation may be important risk factor in the development and expression of the gingival changes. ${ }^{2}$ Treating the excessive tissue enlargement alone without considering other factors such as the drug and local factors such as plaque and calculus will not completely benefit the patient. It is very crucial to include drug substitution and control of local inflammatory factors as initial part of treatment protocol. In this present case the local environmental factors such as poor oral hygiene, the initial presentation may act as risk factors that had contributed to worsen the existing gingival inflammation and further leads to enlargement of gingiva and therefore complicate the oral hygiene procedures. ${ }^{3}$ The importance of oral hygiene in preventing this gingival enlargement should not be underrated as this is probably the best, cheapest and practical option. Surgical correction remains the resort for those who have not responded well to nonsurgical treatment.

\section{Funding}

None.

\section{Acknowledgments}

None.

\section{Conflicts of interest}

Author declares that there are no conflicts of interest. 


\section{References}

1. Lafzi A, Farahani RM, Shoja MA. Phenobarbital-induced gingival hyperplasia. J Contemp Dent Pract. 2007;8(6):50-56.

2. Glickman I, Lewitus M. Hyperplasia of the gingiva associated with Dilantin (sodium diphenyl hydantoinate) therapy. $J$ Am Dent Assoc. $1941 ; 28$.

3. Seymour RA, Thomason JM, Ellis JS

4. . The pathogenesis of drug-induced gingival overgrowth. J Clin Periodontol. 1996;23(3 pt 1):165-175.

5. Merritt H, Putnam T. Sodium diphenylhydantoinate in the treatment of convulsive disorders. J Am Med Assoc. 1938;111(12):1068-1073.

6. Kato $\mathrm{T}$, Okahashi $\mathrm{N}$, Kawai $\mathrm{S}$, et al Impaired degradation of matrix collagen in human gingival fibroblasts by the antiepileptic drug phenytoin. J Periodontol. 2005;76(6):941-950.

7. Kato T, Okahashi N, Ohno T, et al. Effect of phenytoin on collagen accumulation by human gingival fibroblasts exposed to TNF-alpha in vitro. Oral Dis. 2006;12(2):156-162.

8. Goyal L, Bey A, Gupta ND, et al. Diagnosis and management of nonsyndromic hereditary gingival fibromatosis in a 13 year old girl: Report of a rare case. Contemp Clin Dent. 2012;3(Suppl 2):S210-S213.

9. Ilgenli T, Atilla G, Baylas H. Effectiveness of periodontal therapy in patients with drug-induced gingival overgrowth, Long-term results. $J$ Periodontol. 1999;70(9):967-972.

10. Hassell T, Page R, Lindhe J. Histologic evidence for impaired growth control in diphenylhydantoin gingival overgrowth in man. Arch Oral Biol. 1978;23:381-384.

11. May L, Tan E, Holness R, et al. Phenytoin modulates connective tissue metabolism and cell proliferation in human skin fibroblast cultures. Arch Dermatol. 1985;121:79-83.

12. Brunius G, Modéer T. Effect of phenytoin on intracellular $45 \mathrm{Ca} 2+$ accumulation in gingival fibroblasts in vitro. J Oral Pathol Med. 1989;18(8):485-489.

13. Poppell T, Keeling S, Collins F, et al. Effect of folic acid on recurrence of phenytoin-induced gingival overgrowth following gingivectomy. $J$ Clin Periodontol. 1991;18(2):134-139. 